\section{Seroprevalencia de la infección por Trypanosoma cruzi en la población rural del estado Sucre, Venezuela}

\section{Seroprevalence of Trypanosoma cruzi infection in the rural population of Sucre State, Venezuela}

\section{Soroprevalência de infecção pelo Trypanosoma cruzi na população rural do Estado de Sucre, Venezuela}

\author{
Noris García-Jordán 1 \\ Mariolga Berrizbeitia 2,3 \\ Jessicca Rodríguez 2 \\ Juan Luis Concepción 4 \\ Ana Cáceres 4 \\ Wilfredo Quiñones 4
}

doi: 10.1590/0102-311X00050216

\title{
Resumen
}

El objetivo del presente estudio fue determinar la seroprevalencia de la infección por Trypanosoma cruzi en el estado Sucre (Venezuela) y su asociación con factores de riesgo epidemiológicos. El diseño muestral por conglomerados permitió seleccionar 96 centros poblados y 576 viviendas en los 15 municipios del estado. Asimismo, se evaluaron un total de 2.212 muestras de sueros, a través de las pruebas de ELISA, HAI e IFI. La seroprevalencia en el estado Sucre fue de 3,12\%. Los factores de riesgo asociados a la infección por T. cruzi fueron: deposición de basura, materiales predominantes en el piso y paredes, tipo de vivienda, vivir en casas con paredes de bahareque y/o techos de palmas, vivir en casa con paredes y techos de riesgo, construcciones de riesgo $y$ anexos de bahareque, aves dentro de la vivienda y la presencia de leña. La infección se encontró asociada a la edad de los individuos, se detectaron tres casos seropositivos en menores de 15 años. En el estado Sucre existen variables epidemiológicas que favorecen el riesgo a contraer la infección por T. cruzi.

Enfermedad de Chagas; Trypanosoma cruzi; Población Rural

\author{
Correspondencia \\ M. Berrizbeitia \\ Postgrado en Biología Aplicada, Universidad de Oriente. \\ Av. Universidad, Sector San Luis, Cumaná/Sucre - 6101, \\ Venezuela. \\ mberriz@yahoo.com \\ 1 Núcleo de Sucre, Universidad de Oriente, Cumaná, Venezuela. \\ 2 Postgrado en Biología Aplicada, Universidad de Oriente, \\ Cumaná, Venezuela. \\ 3 Instituto de Investigaciones en Biomedicina y Ciencias \\ Aplicadas, Universidad de Oriente, Cumaná, Venezuela. \\ 4 Facultad de Ciencias, Universidad de Los Andes, Mérida, \\ Venezuela.
}




\section{Introducción}

La enfermedad de Chagas es una parasitosis causada por el protozoario hemoflagelado Trypanosoma cruzi, el cual infecta a más de cien especies de animales domésticos y silvestres y puede ser transmitido por aproximadamente 150 especies de insectos hemípteros de la familia Reduviidae, subfamilia Triatominae. Sin embargo, alrededor de 15 géneros pueden transmitir la infección a los humanos, principalmente Triatoma, Panstrongylus y Rhodnius 1 . Se estima que 5,7 millones de personas están infectadas por T. cruzi en 21 países de Latinoamérica y 13\% de la población de esos países se encuentran en riesgo de adquirir la infección. El mayor número de casos reportados para esta parasitosis por transmisión vectorial (activa) ocurren principalmente en Bolivia $(\mathrm{n}=8.087)$, México $(\mathrm{n}=6.135)$ y Colombia $(\mathrm{n}=5.274)^{2}$.

En Venezuela la población que se encuentra en riesgo de adquirir la infección por T. cruzi es de 1.033.450 individuos, se estima que existen 193.339 personas infectadas por el parásito. Cada año ocurren 873 nuevos casos por trasmisión vectorial, la prevalencia estimada es de 0,710\%, mientras que en los bancos de sangre es de 0,320 y la incidencia de la infección por T. cruzi vía congénita por 100 habitantes es $0,110(\mathrm{n}=665)^{2}$.

En Venezuela los estados de mayor seroprevalencia para la infección por T. cruzi son Barinas, Portuguesa, Lara y Trujillo 3 . Estudios recientes muestran prevalencias de 6,85\% en Guariquito (estado Lara), 6,09\% en la comunidad de Copey-El Guayabillo (estado Carabobo), 2,5\% en la comunidad indígena E'nepa (estado Bolívar) y ausencia de individuos infectados en la comunidad La Carolina (estado Bolívar) 4,5,6,7. No obstante, un nuevo escenario epidemiológico aparece en Venezuela para esta parasitosis, cuando en diciembre de 2007, se presenta el primer brote de la infección por T. cruzi transmitida por vía oral, en una escuela del municipio Chacao en Caracas, desde entonces ha ocurrido transmisión por esta vía en 249 individuos (66 adultos y 183 niños) con 10 fallecimientos en diferentes localidades del país 8 .

En relación a la situación de la enfermedad de Chagas, y los factores de riesgo epidemiológicos asociados a esta patología, son pocos los estudios realizados en el oriente del país, si se compara con los realizados en la región centro-occidental de Venezuela. Entre los factores de riesgo epidemiológicos asociados con la infestación triatomínica se encuentran el número de residentes de la vivienda, la presencia de troja en el peridomicilio y la falta de rociamiento con insecticidas, mientras que entre los asociados a la seropositivad humana se encuentran, la edad, la presencia intradomiciliaria del vector, viviendas con piso de tierra, el consumo de chimó, la presencia en forma temporal o permanente de perros, caprinos, armadillos y aves en el domicilio, el desorden y la presencia de basura en el domicilio, y en el peridomicilio la presencia de anexos de bahareque, entre otros 9,10. Más recientemente, en un estudio realizado en diferentes localidades rurales del estado Lara, los factores de riesgo que se encontraron asociados a la enfermedad de Chagas fueron la presencia del vector en la vivienda, comer animales de caza, la migración rural y contacto con el vector 4 .

Es de suma importancia conocer la situación de la infección por T. cruzi, especialmente, en regiones poco estudiadas en Venezuela. Por tal motivo, el objetivo de este trabajo fue determinar la seroprevalencia y los factores de riesgo asociados a la infección por T. cruzi en individuos que habitan en 96 centros poblados rurales, ubicados en los 15 municipios que forman el estado Sucre de Venezuela.

\section{Métodos}

\section{Área de estudio}

El estudio se realizó en el estado Sucre de Venezuela entre las coordenadas geográficas 10³8’44” de latitud norte y 6302'20” de longitud oeste, en el período comprendido de agosto 2008 a noviembre de 2008, lo cual coincidió con la estación lluviosa en Venezuela. Este estado se encuentra ubicado en la región Nororiental de Venezuela. El estado Sucre está compuesto por los municipios: Andrés Eloy Blanco, Andrés Mata, Arismendi, Benítez, Bermúdez, Bolívar, Cajigal, Cruz Salmerón Acosta, Libertador, Mariño, Mejía, Montes, Ribero, Sucre y Valdez. 


\section{Selección de la muestra y organización del estudio}

Para el marco muestral de la zona de estudio se contó con los directorios y cartografía, proporcionados por el Instituto Nacional de Estadística (INE) en el Censo 2001, y actualizados por el mismo organismo para el año 2008. El tamaño de la muestra se calculó de manera que fuera válido tanto para la población de viviendas, como para la población humana, en cada una de las áreas de estudio (Tabla 1). Tomando niveles de confianza iguales o superiores a 95\% y como límite máximo del error de estimación el 2\%, y asumiendo una prevalencia del $10 \%$.

Se realizó un estudio de corte transversal con un muestreo probabilístico aleatorio, en el cual se recurrió a un diseño por conglomerados bietápico, en el cual las unidades primarias (centros poblados) se eligieron proporcionales al tamaño del municipio, utilizando un esquema aleatorio simple sin reemplazo, y las viviendas fueron seleccionadas de acuerdo a uno sistemático con origen aleatorio, cuyo tamaño fue prefijado en seis viviendas por unidad primaria 11. Aplicando esta metodología se seleccionaron de manera aleatoria 96 centros poblados y 576 viviendas. Los criterios de inclusión de los habitantes de las viviendas que resultaron seleccionadas fueron: residentes permanentes, individuos de ambos sexos con edades mayores al año de edad.

Para este estudio se realizaron dos visitas, en la primera un sociólogo se encargó de la localización de las viviendas, contacto con organismos públicos y líderes de las comunidades dispuestos a colaborar, corrección de los mapas (croquis) con las viviendas a evaluar, sensibilización en los centros poblados sobre los objetivos del proyecto y la importancia y los beneficios que el estudio traería a las comunidades. En la segunda visita se aplicaron las encuestas epidemiológicas, consentimiento informado y se realizó la toma de muestras serológicas, por profesionales del área (bioanalistas y/o enfermeras), a las personas residentes de las viviendas seleccionadas.

Tabla 1

Distribución de los centros poblados rurales, viviendas y población rural estimada para el año 2008, y muestras evaluadas para la infección por Trypanosoma cruzi en el estado Sucre, Venezuela.

\begin{tabular}{|c|c|c|c|c|c|c|c|}
\hline Municipios & $\begin{array}{l}\text { Centros } \\
\text { poblados } \\
\text { rurales }\end{array}$ & Peso & $\begin{array}{c}\text { Centros } \\
\text { poblados } \\
\text { muestreados }\end{array}$ & $\begin{array}{l}\text { Viviendas } \\
\text { rurales } \\
\text { totales }\end{array}$ & $\begin{array}{c}\text { Viviendas rurales } \\
\text { muestreadas }\end{array}$ & $\begin{array}{l}\text { Población } \\
\text { rural }\end{array}$ & $\begin{array}{l}\text { Población } \\
\text { muestreada }\end{array}$ \\
\hline Andrés Eloy Blanco & 16 & 0,02 & 2 & 1.631 & 11 & 5.171 & 53 \\
\hline Andrés Mata & 23 & 0,04 & 3 & 1.985 & 20 & 8.421 & 60 \\
\hline Arismendi & 76 & 0,12 & 11 & 4.899 & 67 & 16.187 & 243 \\
\hline Benítez & 93 & 0,09 & 9 & 5.758 & 53 & 21.525 & 232 \\
\hline Bermúdez & 12 & 0,02 & 2 & 1.437 & 11 & 4.928 & 38 \\
\hline Bolívar & 27 & 0,03 & 3 & 1.638 & 18 & 4.426 & 52 \\
\hline Cajigal & 42 & 0,04 & 4 & 2.126 & 23 & 7.381 & 88 \\
\hline Cruz Salmerón & 33 & 0,07 & 6 & 2.772 & 38 & 10.948 & 168 \\
\hline \multicolumn{8}{|l|}{ Acosta } \\
\hline Libertador & 11 & 0,03 & 3 & 1.166 & 16 & 4.073 & 76 \\
\hline Mariño & 44 & 0,10 & 10 & 4.289 & 59 & 13.881 & 217 \\
\hline Mejía & 39 & 0,06 & 5 & 2.293 & 32 & 8.543 & 103 \\
\hline Montes & 121 & 0,12 & 12 & 5.096 & 69 & 18.059 & 286 \\
\hline Ribero & 95 & 0,14 & 14 & 6.895 & 81 & 23.935 & 350 \\
\hline Sucre & 83 & 0,07 & 6 & 4.461 & 38 & 16.049 & 119 \\
\hline Valdez & 38 & 0,06 & 6 & 2.405 & 32 & 7.758 & 127 \\
\hline Total & 753 & 1,00 & 96 & 48.851 & 576 & 171.285 & 2.212 \\
\hline
\end{tabular}




\section{Recolección de las muestras serológicas}

Las muestras de sangre fueron obtenidas por venopunción, se extrajeron $5 \mathrm{~mL}$ a cada uno de los individuos de manera voluntaria, éstas fueron transportadas a $4^{\circ} \mathrm{C}$ al laboratorio y centrifugadas a $1.000 \mathrm{~g}$ por 10 minutos a temperatura ambiente y los sueros fueron almacenados a $-70^{\circ} \mathrm{C}$ hasta su posterior uso.

\section{Análisis serológico de las muestras sanguíneas}

Para la detección de anticuerpos anti-T. cruzi en las muestras de los sueros evaluados, se emplearon las técnicas de ensayo inmunoenzimático indirecto (ELISA), utilizando epimastigotes fijados 12 y la prueba de hemaglutinación indirecta (HAI) (Wiener Laboratorios S.A.I.C., Rosario, Argentina). Esta última se efectuó para confirmar todas las muestras que resultaran positivas para infección por T. cruzi por la prueba de ELISA, y en el caso de resultados inconclusos, se aplicó una tercera técnica (inmunofluorescencia indirecta -IFI) 13. Los sueros de los individuos se consideraron positivos, cuando estos fueron reactivos con dos de las tres técnicas utilizadas.

\section{Prueba de ELISA utilizando epimastigotes fijados}

Para la realización de esta prueba se utilizaron epimastigotes fijados de una mezcla 1:1 de cepas de referencia internacional de T. cruzi (cepa Tulahuen y Brasil), siguiendo el protocolo descrito por Berrizbeitia et al. 12. Todos los sueros fueron evaluados por duplicado y en experimentos independientes. Una mezcla de sueros controles positivos y negativos, confirmados por tres pruebas serológicas diferentes en el Laboratorio de Inmunodiagnóstico de Chagas (Maracay, Venezuela), fueron incluidos en cada placa. Los resultados fueron aceptados sólo si el coeficiente de variación (CV) para cada placa y entre placas fue menor o igual a 15\%; de otro modo las muestras fueron analizadas nuevamente. El punto de corte para esta prueba fue determinado utilizando la curva: "receiver-operating characteristic curves". Esta curva definió el valor de 0,400 de densidad óptica (DO) como el valor óptimo, el cual permitió la discriminación entre valores positivos y negativos 12 .

\section{Determinación de los factores de riesgo y análisis de los datos}

Para la determinación de los factores de riesgo se aplicaron a los jefes de hogar encuestas epidemiológicas, las cuales fueron completadas con la observación directa de las variables estudiadas en cada una de las viviendas seleccionadas. Las encuestas evaluaron las siguientes características: individuales (edad, sexo, haber habitado en viviendas con paredes de bahareque, techo o paredes de palma), factores domiciliarios (tipo de vivienda, materiales predominantes en el techo, paredes y piso, presencia de animales domésticos y silvestres en el interior de la vivienda) y factores peridomiciliarios (plantaciones de palma, anexos de bahareque, y presencia de animales silvestres y domésticos, depósitos de herramientas), conocimiento de la enfermedad de Chagas y conocimiento de los triatominos.

\section{Análisis estadístico}

Para establecer la relación existente entre los posibles factores de riesgo epidemiológico con las casas donde habitaba al menos un individuo seropositivo a la infección por T. cruzi, se aplicó la prueba de chi al cuadrado $\left(\chi^{2}\right)$ con corrección de Jates. También, se determinaron otros índices seroepidemiológicos: infección por T. cruzi, densidad de infección, y dispersión en viviendas y en centros poblados rurales del estado Sucre. La razón de odds (del inglés, odds ratio, OR), con un intervalo de 95\% de confianza (IC95\%) y un nivel de significancia de 0,05, se utilizó para determinar el efecto independiente de las asociaciones 14 . Las variables no significativas se eliminaron de forma progresiva paso a paso y se aplicó un análisis de regresión logística binaria. Se utilizaron los programas de computador Excel 2010 (Microsoft Corp., EE.UU.), SPSS, versión 18 (IBM Corp., Armonk, EE.UU.), Statgraphics (http:// www.statgraphics.com/), para el procesamiento y análisis estadístico de los datos. 


\section{Consideraciones éticas}

El trabajo de campo se realizó con un equipo conformado por dos sociólogos, un bioanalista, un biólogo y los líderes comunitarios de cada centro poblado para informar a la población, aplicación de las encuestas epidemiológicas, toma de las muestras de sangre de los individuos y supervisión. Las muestras de sangre fueron tomadas después de que los jefes de familia y los habitantes de las viviendas aceptaran de forma voluntaria, firmando el consentimiento informado. El protocolo del estudio fue revisado y aprobado por la Comisión Nacional de Bioética y Bioseguridad en Salud del Ministerio del Poder Popular para la Salud (MPPS) de Venezuela.

\section{Resultados}

\section{Diagnóstico serológico}

De los 2.212 individuos muestreados, el 53,35\% ( $\mathrm{n}=1.180)$ fueron del género femenino, mientras que el 46,65\% ( $n=1.032)$ del género masculino. El promedio de la edad de los individuos fue 30,25 años \pm 21,89 (rango: 1-95 años). De los 2.212 sueros analizados con la prueba de ELISA, 78/2212 resultaron positivos para la infección por T. cruzi, de éstos 66/78 fueron confirmados como positivos por HAI, teniendo 12 individuos con resultados inconclusos (positivos por ELISA y negativos por HAI). Los 12 sueros fueron evaluados por IFI y $3 / 12$ de los sueros inconclusos fueron confirmados como positivos. Por lo tanto, la seroprevalencia de la infección por T. cruzi en la población evaluada, utilizando como criterio el resultado positivo de dos pruebas serológicas, fue de 3,12\% (69/2.212).

Se observó asociación estadística significativa entre la infección por T. cruzi y la edad de los individuos, encontrándose mayores seroprevalencias a partir de los 40 años de edad $\left(\chi^{2}: 195,8\right.$ grados de libertad, $\mathrm{p}=0,0001)$. Asimismo, tres individuos menores de 10 años $(0,14 \%)$ fueron seropositivos y uno de ellos tenía una edad de 2 años. Aunque el género femenino presentó un mayor número de seropositivos $(\mathrm{n}=39)$ con respecto al masculino $(\mathrm{n}=30)$, éste no estuvo asociado a la infección por $T$. cruzi $(\mathrm{p}>0,05)$ (Tabla 2).

Tabla 2

Seroprevalencia de la infección por Trypanosoma cruzi, según la edad y el género en la población rural del estado Sucre, Venezuela.

\begin{tabular}{|c|c|c|c|c|c|c|c|c|}
\hline \multirow[t]{2}{*}{ Rango de edad (años) } & \multicolumn{2}{|c|}{ Negativos } & \multicolumn{2}{|c|}{ Positivos } & \multirow[t]{2}{*}{ Total } & \multirow[t]{2}{*}{ PG (\%) } & \multirow[t]{2}{*}{ PE (\%) } & \multirow[t]{2}{*}{$\mathbf{p}$} \\
\hline & Masculino & Femenino & Masculino & Femenino & & & & \\
\hline $1-10$ & 246 & 205 & 1 & 2 & 454 & 0,14 & 0,66 & \\
\hline $11-20$ & 233 & 286 & 0 & 0 & 519 & 0,00 & 0,00 & \\
\hline $21-30$ & 124 & 175 & 1 & 0 & 300 & 0,05 & 0,33 & \\
\hline $31-40$ & 112 & 137 & 1 & 3 & 253 & 0,18 & 1,58 & 0,0001 \\
\hline $41-50$ & 92 & 130 & 5 & 4 & 231 & 0,41 & 3,90 & \\
\hline $51-60$ & 79 & 92 & 7 & 10 & 188 & 0,77 & 9,04 & \\
\hline $61-70$ & 54 & 56 & 10 & 5 & 125 & 0,68 & 12,00 & \\
\hline $71-80$ & 40 & 44 & 3 & 11 & 98 & 0,63 & 14,29 & \\
\hline $81-90$ & 15 & 16 & 1 & 3 & 35 & 0,18 & 11,43 & \\
\hline $91-95$ & 1 & 0 & 1 & 0 & 2 & 0,05 & 50,00 & \\
\hline Sin edad registrada & 6 & 0 & 0 & 1 & 7 & 0,05 & 14,29 & \\
\hline Total & 1.002 & 1.141 & 30 & 39 & 2.212 & 3,12 & - & \\
\hline
\end{tabular}

p: probabilidad; PE: prevalencia específica (porcentaje de seropositivos de cada grupo por edad, respecto a la población de cada grupo etario); PG: prevalencia general (porcentaje de seropositividad de cada grupo por edad, respecto a la población total).

Nota: prueba de $\chi^{2}$ : 146; 8 grados de libertad; $p<0,05$ : significativo. 
La seroprevalencia de anticuerpos anti-T. cruzi, según la localización geográfica de los municipios, se estratificó en dos regiones geográficas. Para la parte oeste del estado Sucre se ubican los municipios pertenecientes al golfo de Cariaco y para el este aquellos del golfo de Paria (Figura 1). La seroprevalencia general para el golfo de Cariaco fue de $2,85 \%$, la cual fue superior a la registrada en el golfo de Paria (0,27\%). Para ambas regiones geográficas el mayor número de seropositivos se presentó en los individuos mayores de 40 años y en el género femenino (Tabla 3). No se encontraron individuos seropositivos en los municipios Andrés Mata, Benítez, Bermúdez, Cruz Salmerón Acosta y Mariño. La prueba de $\chi^{2}$ mostró diferencias estadísticas significativas entre estas dos regiones geográficas y la infección por T. cruzi. Lo que indica un mayor número de individuos seropositivos para los que habitan en los municipios ubicados en el golfo de Cariaco $\left(\chi^{2}=23,89\right.$; grados de libertad: $\left.1 ; \mathrm{p}<0,05\right)$, indicando que habitar en esta región geográfica aumenta siete veces el riesgo de contraer la infección por T. cruzi (OR = 7,46; IC95\%: 2,60-11,46) (Tabla 3). Asimismo, se encontraron individuos seropositivos menores de 10 años en ambas regiones geográficas, dos en la región del golfo de Cariaco y uno en la región del golfo de Paria (Tabla 3).
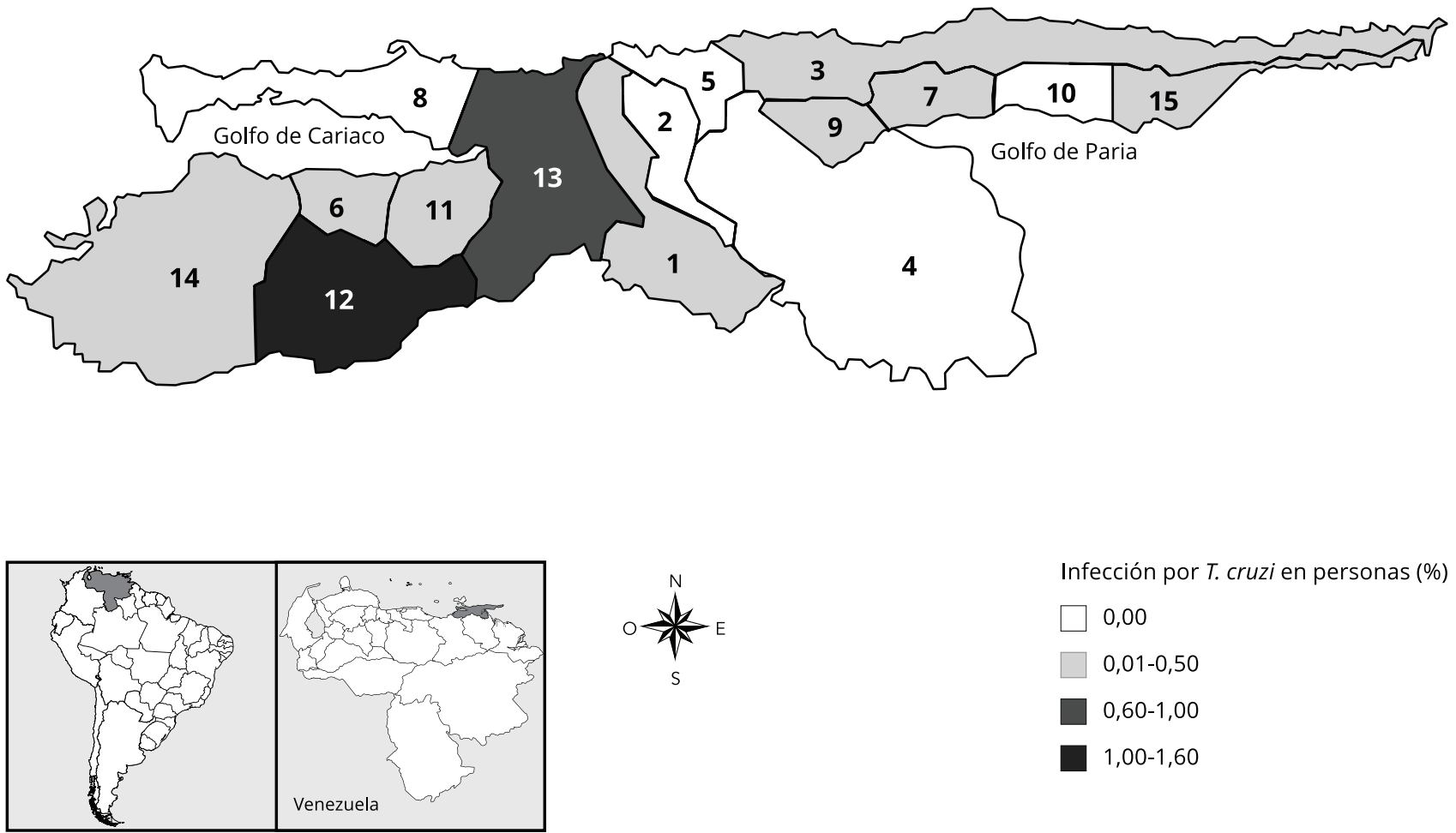

Infección por T. cruzi en personas (\%)

0,00

$0,01-0,50$

$0,60-1,00$

$1,00-1,60$

Nota: municipios pertenecientes al golfo de Cariaco: 6 (Bolívar), 8 (Cruz Salmerón Acosta), 11 (Mejía), 12 (Montes), 13 (Ribero) y 14 (Sucre); municipios pertenecientes al golfo de Paria: 1 (Andrés Eloy Blanco), 2 (Andrés Mata), 3 (Arismendi), 4 (Benítez), 5 (Bermúdez), 7 (Cajigal), 9 (Libertador), 10 (Mariño) y 15 (Valdez). 


\section{Tabla 3}

Seroprevalencia de anticuerpos anti-Trypanosoma cruzi, según el grupo etario y sexo en la población rural del estado Sucre, Venezuela, de acuerdo a la ubicación geográfica de los municipios.

\begin{tabular}{|c|c|c|c|c|c|c|c|c|c|}
\hline \multirow[t]{2}{*}{ Rango de edad (años) } & \multicolumn{3}{|c|}{ Positivos } & \multicolumn{3}{|c|}{ Negativos } & \multirow[t]{2}{*}{ Total } & \multirow[t]{2}{*}{ PG } & \multirow[t]{2}{*}{ IC95\% } \\
\hline & Masculino & Femenino & Total & Masculino & Femenino & Total & & & \\
\hline \multicolumn{10}{|c|}{ Municipios del golfo de Cariaco } \\
\hline $1-10$ & 1 & 1 & 2 & 136 & 118 & 254 & 256 & \multirow{12}{*}{2,85} & \multirow{12}{*}{$2,16-3,54$} \\
\hline $11-20$ & 0 & 0 & 0 & 130 & 173 & 303 & 303 & & \\
\hline $21-30$ & 1 & 0 & 1 & 85 & 101 & 186 & 187 & & \\
\hline $31-40$ & 0 & 2 & 2 & 73 & 89 & 162 & 164 & & \\
\hline $41-50$ & 5 & 3 & 8 & 50 & 76 & 126 & 134 & & \\
\hline $51-60$ & 7 & 9 & 16 & 45 & 55 & 100 & 116 & & \\
\hline $61-70$ & 10 & 5 & 15 & 32 & 31 & 63 & 78 & & \\
\hline $71-80$ & 3 & 11 & 14 & 18 & 23 & 41 & 55 & & \\
\hline $81-90$ & 1 & 2 & 3 & 8 & 4 & 12 & 15 & & \\
\hline $91-95$ & 1 & 0 & 1 & 1 & 0 & 1 & 2 & & \\
\hline Sin edad registrada & 0 & 1 & 1 & 0 & 0 & 5 & 6 & & \\
\hline Total & 29 & 34 & 63 & 578 & 670 & 1.253 & 1.316 & & \\
\hline \multicolumn{10}{|c|}{ Municipios del golfo de Paria } \\
\hline $1-10$ & 0 & 1 & 1 & 110 & 96 & 206 & 207 & \multirow{12}{*}{0,27} & \multirow{12}{*}{$0,00-0,49$} \\
\hline $11-20$ & 0 & 0 & 0 & 103 & 113 & 216 & 216 & & \\
\hline $21-30$ & 0 & 0 & 0 & 39 & 65 & 104 & 104 & & \\
\hline $31-40$ & 1 & 1 & 2 & 39 & 48 & 87 & 89 & & \\
\hline $41-50$ & 0 & 1 & 1 & 42 & 54 & 96 & 97 & & \\
\hline $51-60$ & 0 & 1 & 1 & 34 & 37 & 71 & 72 & & \\
\hline $61-70$ & 0 & 0 & 0 & 22 & 25 & 47 & 47 & & \\
\hline $71-80$ & 0 & 0 & 0 & 22 & 21 & 43 & 43 & & \\
\hline $81-90$ & 0 & 1 & 1 & 7 & 12 & 19 & 20 & & \\
\hline $91-95$ & 0 & 0 & 0 & 0 & 0 & 0 & 0 & & \\
\hline Sin edad registrada & 0 & 0 & 0 & 1 & 0 & 1 & 1 & & \\
\hline Total & 1 & 5 & 6 & 419 & 471 & 890 & 896 & & \\
\hline
\end{tabular}

IC95\%: intervalo del 95\% de confianza; PG: prevalencia general.

Nota: $\chi^{2}: 23,89 ; p<0,0001$; razón de probabilidades: 7,46 (IC95\%: 2,60-11,46).

\section{Índices seroepidemiológicos}

Se encontraron individuos positivos a la infección por $T$. cruzi en 10/15 (66,67\%) municipios que conforman el estado Sucre, el número de centros poblados con personas seropositivas fue de 33/96 (dispersión en centros poblados: 34,38\%). Igualmente, se encontraron 55/576 viviendas, donde al menos uno de sus residentes resultó seropositivo a la infección (dispersión en vivienda y de densidad 9,55\% y 0,12, respectivamente). Los municipios que presentaron las mayores seroprevalencias para la infección por T. cruzi fueron Montes (1,31\%; IC95\%: 0,84-1,78), seguido de Ribero (0,81\%; IC95\%: 0,44-1,18) y Sucre (0,45\%; IC95\%: 0,17-0,73) (Tabla 4). Mientras que los dos centros poblados rurales del estado Sucre con mayores seroprevalencias fueron: El Potrero (0,27\%; IC95\%: 0,04-2,76, municipio Montes) y Las Calderas (0,23\%; IC95\%: 0,23-3,27, municipio Montes).

\section{Factores de riesgo asociados a la infección por $T$. cruzi}

Para evaluar los factores de riesgo se clasificaron las viviendas como positivas si al menos uno de los individuos era seropositivo a la infección por T. cruzi. Los resultados del análisis univariado (prueba de $\chi^{2}$ ), obtenidos de los datos arrojados a través de las encuestas realizadas a la población rural del 
Tabla 4

Seroprevalencia de la infección por Trypanosoma cruzi en los municipios donde se encontraron casos positivos de la población rural del estado Sucre, Venezuela.

\begin{tabular}{lccccccc}
\hline Municipio & \multicolumn{2}{c}{ Positivos } & \multicolumn{2}{c}{ Negativos } & Total & PG (\%) & IC95\% \\
& Masculino & Femenino & Masculino & Femenino & & \\
\hline Andreś Eloy Blanco & 0 & 1 & 25 & 27 & 53 & 0,05 & $0,00-0,13$ \\
Arismendi & 0 & 1 & 125 & 117 & 243 & 0,05 & $0,00-0,13$ \\
Bolívar & 0 & 1 & 21 & 30 & 52 & 0,05 & $0,00-0,13$ \\
Cajigal & 0 & 2 & 45 & 41 & 88 & 0,09 & $0,00-0,22$ \\
Libertador & 1 & 0 & 32 & 43 & 76 & 0,05 & $0,00-0,13$ \\
Mejías & 2 & 3 & 42 & 56 & 103 & 0,23 & $0,02-0,42$ \\
Montes & 11 & 18 & 111 & 146 & 286 & 1,31 & $0,84-1,79$ \\
Ribero & 14 & 4 & 150 & 182 & 350 & 0,81 & $0,44-1,19$ \\
Sucre & 2 & 8 & 47 & 62 & 119 & 0,45 & $0,17-0,73$ \\
Valdez & 0 & 1 & 57 & 69 & 127 & 0,05 & $0,00-0,13$ \\
Total & 30 & 39 & 655 & 773 & 1.497 & 3,12 & $2,30-3,84$ \\
\hline
\end{tabular}

IC95\%: intervalo del 95\% de confianza; PG: prevalencia general.

Nota: se muestran sólo los municipios con casos seropositivos.

estado Sucre, se muestran en la Tabla 5. Ésta muestra sólo las variables que resultaron asociadas a la infección por T. cruzi $(\mathrm{p}<0,05)$. Esas variables se asociaron significativamente con la seroprevalencia por el análisis univariado, sin embargo, sólo la basura que se entierra y se quema siguieron siendo significativas en el modelo multivariado. Las variables epidemiológicas que no presentaron asociación estadística significativa con la infección por T. cruzi fueron: el género, la ocupación de los individuos, el conocimiento acerca de la enfermedad de Chagas, tiempo de residencia en la vivienda, el conocimiento y reconocimiento de los triatominos. Entre los factores domiciliarios: la presencia de animales domésticos y entre los factores peridomiciliarios: la presencia de palmas, corrales, presencia de animales domésticos y silvestres en el peridomicilio ( $\mathrm{p}>0,05)$.

\section{Discusión}

Este trabajo representa un extenso estudio realizado en una amplia zona geográfica del estado Sucre, ubicado en la región nororiental de Venezuela, en donde se abarcó sus 15 municipios y de forma representativa y aleatoria sus localidades rurales y habitantes. La seroprevalencia de la infección para T. cruzi en esta área geográfica fue de $3,12 \%$, la cual es baja cuando se compara con los resultados obtenidos en el occidente del país, con una seroprevalencia general en Venezuela de un 11\%, en 4.000 muestras analizadas en 75 localidades endémicas de 10 estados del territorio nacional 15. Mientras que en otros trabajos realizados en el oriente del país en localidades de los estados Sucre, Anzoátegui, Monagas y Bolívar las seroprevalencias varían de 0 a 9\% 7,16,17,18,19. Un aspecto muy relevante de la presente investigación es la detección de casos seropositivos en menores de 10 años, debido a que éstos no recibieron transfusión sanguínea y sus madres fueron seronegativas se descartó la transmisión congénita y transfusional. Este hallazgo demuestra que existe una transmisión vectorial activa muy pequeña en ambas regiones geográficas del estado Sucre (municipios del golfo de Cariaco y Paria). La baja seroprevalencia en niños encontrada en este estudio $(0,14 \%)$ es similar a la encontrada para 3.296 infantes en el estado Barinas, con seroprevalencia para T. cruzi de 0,12\%, mientras que en el estado Lara (municipio Andrés Eloy Blanco), se reportó una seroprevalencia en niños menores de 10 años de $0,57 \% 10,20$.

Los resultados en relación a los valores obtenidos de seroprevalencia podrían atribuirse a que el principal vector descrito en el estado Sucre lo constituye T. maculata 21, este triatomino se encuentra 
Tabla 5

Factores de riesgo asociados a la infección por Trypanosoma cruzi en los diferentes municipios de la población rural del estado Sucre, Venezuela.

\begin{tabular}{|c|c|c|c|c|c|}
\hline Factor de riesgo & Viviendas & $\begin{array}{l}\text { Positivos para el factor de riesgo } \\
\qquad[\%(n)]\end{array}$ & OR & IC95\% & $\mathbf{p}$ \\
\hline \multicolumn{6}{|l|}{ Enterrar la basura } \\
\hline Sí & 30 & $23,33(7)$ & 3,2 & $1,29-7,74$ & 0,008 \\
\hline No & 546 & $8,79(48)$ & & & \\
\hline \multicolumn{6}{|l|}{ Quemar la basura } \\
\hline Sí & 384 & $7,55(29)$ & 0,52 & $0,30-0,91$ & 0,03 \\
\hline No & 192 & $13,54(26)$ & & & \\
\hline \multicolumn{6}{|c|}{ Materiales predominantes de la pared } \\
\hline Primera & 89 & $22,47(20)$ & 3,74 & $2,04-6,85$ & 0,000 \\
\hline Segunda & 487 & $7,19(35)$ & & & \\
\hline \multicolumn{6}{|c|}{ Materiales predominantes del piso } \\
\hline Tierra & 92 & $7,61(7)$ & - & - & 0,000 \\
\hline Cemento & 395 & $7,09(28)$ & & & \\
\hline Otros & 89 & $22,47(20)$ & & & \\
\hline \multicolumn{6}{|l|}{ Tipo de vivienda } \\
\hline Rancho & 94 & $17,02(16)$ & 2,33 & $1,24-4,37$ & 0,01 \\
\hline Casa & 482 & $8,09(39)$ & & & \\
\hline \multicolumn{6}{|c|}{ Haber habitado en casa con paredes o techos de palmas } \\
\hline Sí & 411 & $11,68(48)$ & 2,98 & $1,32-6,74$ & 0,00 \\
\hline No & 165 & $4,24(7)$ & & & \\
\hline \multicolumn{6}{|c|}{ Haber habitado en casa con paredes de bahareque } \\
\hline $\mathrm{Ns} / \mathrm{Nc}$ & 44 & $6,82(3)$ & - & - & 0,021 \\
\hline Sí & 181 & $4,97(9)$ & & & \\
\hline No & 351 & $12,25(43)$ & & & \\
\hline \multicolumn{6}{|l|}{ Paredes de riesgo } \\
\hline Sí & 355 & $11,55(41)$ & 1,93 & $1,02-3,63$ & 0,05 \\
\hline No & 221 & $6,33(14)$ & & & \\
\hline \multicolumn{6}{|l|}{ Techos de riesgo } \\
\hline Sí & 345 & $11,59(40)$ & 1,89 & $1,01-3,5$ & 0,05 \\
\hline No & 231 & $6,49(15)$ & & & \\
\hline \multicolumn{6}{|c|}{ Depósitos de herramientas } \\
\hline Sí & 382 & $11,52(44)$ & 2,16 & $1,09-4,29$ & 0,03 \\
\hline No & 194 & $5,67(11)$ & & & \\
\hline \multicolumn{6}{|l|}{ Anexos de bareque } \\
\hline Sí & 145 & $17,24(25)$ & 2,78 & $1,58-4,92$ & 0,00 \\
\hline No & 431 & $6,96(30)$ & & & \\
\hline \multicolumn{6}{|c|}{ Aves dentro de la vivienda } \\
\hline Sí & 136 & $14,70(20)$ & 2,00 & $1,11-3,59$ & 0,03 \\
\hline No & 440 & $8,75(35)$ & & & \\
\hline \multicolumn{6}{|l|}{ Leña en la vivienda } \\
\hline Sí & 354 & $11,86(42)$ & 2,16 & $1,13-4,13$ & 0,02 \\
\hline No & 222 & $5,86(13)$ & & & \\
\hline
\end{tabular}

IC95\%: intervalo del 95\% de confianza; Ns/Nc: no sabe/no constesta; OR: odds ratio. Nota: $p<0,05$ (significativo). 
en regiones xerófilas y costeras de Venezuela, además es considerado una especie ornitófoga, asociado a las aves en el hábitat peridoméstico, esta característica lo condiciona a tener una capacidad vectorial limitada, debido a que presenta bajos índices de infección por sus fuentes de alimento 4,22. En los estados occidentales, donde se reporta una mayor endemicidad de la enfermedad de Chagas, el vector principal lo constituye $R$. prolixus 23,24 .

En el presente trabajo se encontraron individuos seropositivos en el 66,67\% de los municipios, $34,38 \%$ de los centros poblados y 9,55\% de las viviendas rurales del estado Sucre. Por otra parte, los resultados de este estudio confirman que la edad está significativamente asociada a la seropositividad por T. cruzi, el grupo etario con el mayor número de casos positivos fue entre 40 y 80 años. Estos resultados demuestran que la mayoría de los casos seropositivos, reflejan un problema de exposición antigua, debido a que antes de 1950 aún no existían en Venezuela campañas de fumigación en las viviendas o en sectores rurales o semirurales, por lo cual el contacto vectorial era superior que en la actualidad 25 .

Cuando evaluamos la seropositividad a la infección por T. cruzi encontramos diferencias estadísticas significativas entre las regiones geográficas evaluadas ( $<$ 0,05) (golfo de Cariaco y Paria). El mayor número de individuos seropositivos se encontró en los municipios pertenecientes a la región del golfo de Cariaco. La enfermedad de Chagas predomina en regiones con características geográficas tales como: zonas cafetaleras, pie de monte y variables climáticas como de temperaturas que oscilan entre $19^{\circ} \mathrm{C}$ y $25^{\circ} \mathrm{C}$ y $75 \%$ de humedad relativa, además su distribución abarca desde el nivel del mar hasta los $2.000 \mathrm{~m}$ sobre éste 25 . Las características geográficas, climáticas y topográficas de los municipios ubicados en golfo de Cariaco 26 favorecen la transmisión de la infección por T. cruzi, lo cual quedó evidenciado al encontrarse un mayor número de casos seropositivos en los centros poblados de esos municipios. Sin embargo, las características geográficas y climatológicas como temperaturas entre $25^{\circ} \mathrm{C}$ y $34^{\circ} \mathrm{C}$, humedad relativa entre 80 a $90 \%$ y la topografía comprendida entre 200 y $800 \mathrm{~m}$ de altitud sobre el nivel del mar (msnm), para los municipios ubicados en el golfo de Paria 26, no son las más adecuadas para mantener la cadena epidemiológica, tanto del vector, como del parásito. Es por ello que en algunos municipios ubicados en el golfo de Paria no se encontraron individuos seropositivos, debido a que los períodos constantes de pluviosidad y alta humedad presentes en esa zona, afecta considerablemente la reproducción y el nicho ideal para los vectores triatominos 27.

En un estudio entomológico, realizado en el estado Sucre y publicado previamente por nuestro grupo de investigación, se describe que el vector principal en el estado Sucre es T. maculata y la infección natural triatomínica fue de 1,72\%. Igualmente, se reportó que los municipios y viviendas con mayor índice de infestación por triatominos fueron Mariño (60\%), Benítez y Libertador (33,33\%), todos pertenecientes a la zona geográfica de Paria 21, lo cual contrasta con los resultados de la serología en humanos, donde los mayores índices de infección por T. cruzi fueron detectados en la región del golfo de Cariaco. Todos estos hallazgos nos permiten plantear que la mayor seroprevalencia de T. cruzi, encontrada principalmente en la zona geográfica del golfo de Cariaco, se debe a infecciones antiguas, y fue en esa región donde posiblemente se enfocaron en el pasado las campañas de fumigación para el control vectorial.

Los factores de riesgos asociados a la infección por T. cruzi en esta investigación son los primeros reportes para el estado Sucre, razón por la cual no pueden hacerse estudios comparativos de estos resultados con décadas pasadas. En relación a estas variables epidemiológicas evaluadas, se demostró una asociación estadística significativa en el modo final de deposición de la basura, en las poblaciones evaluadas, y la infección por T. cruzi. Se ha demostrado que la basura potencia el riesgo de contraer la infección, debido a que ésta favorece los criaderos de ratas, ratones y rabipelados los cuales son reservorios de T. cruzi 9. Quemar la basura resultó un factor protector para adquirir la infección, ya que de esta forma se elimina la probabilidad de establecer un espacio que permita la presencia y reproducción de los triatominos. Mientras que enterrar la basura resulta ser un factor que aumenta la posibilidad de adquirir la enfermedad, a pesar de que este método de deposición final de la basura provoca que los insectos triatominos pierdan lugares de refugio y fuentes inmediatas de alimento, es posible que las personas encuestadas suministraron información falsa o errónea acerca de la deposición final de la basura. Por lo cual es fácil de suponer que la basura no sea totalmente enterrada, sino que queda expuesta o a la intemperie, lo que proporcione un refugio a los insectos para reproducirse y colonizar la vivienda por largo tiempo, aumentando la probabilidad de la transmisión vectorial. Al igual 
que en la presente investigación en un estudio realizado en el municipio Urdaneta (estado Lara) por Bonfante-Cabarcas et al. ${ }^{9}$ se demostró una asociación positiva entre el acúmulo de artefactos viejos, desechos, materiales de construcción, basura y la limpieza inadecuada de la vivienda y la infección por T. cruzi.

Al analizar la relación de la seropositividad a T. cruzi y las características de las viviendas evaluadas se encontró asociación significativa entre la infección y los materiales de construcción de las viviendas. Estos resultados coinciden por lo reportado en trabajos epidemiológicos realizados en los estados Lara (municipio Urdaneta) y Barinas en los cuales se determinó que existe asociación significativa entre las características de los materiales de construcción de las viviendas y la seropositividad a T. cruzi 9,10. Las viviendas con paredes sin frisar, con muchas grietas en pisos y techos, que están construidas con materiales de desecho y que se encuentran cerca de árboles o en zonas boscosas, con ratas o rabipelados, son más propensas a la proliferación de triatominos en el interior o en exterior de la vivienda 28 .

Al analizar el tipo de vivienda y la seropositividad a $T$. cruzi se demostró en la presente investigación que habitar en una vivienda, clasificada como rancho, está asociado a un mayor riesgo de adquirir la infección por el parásito. Es conocido que la enfermedad de Chagas se considera como un problema asociado a viviendas de bahareque y paja (ranchos), debido a la facilidad de los insectos triatominos para infestarlas, reproducirse y mantener la cadena del parásito circulando en la vivienda 28 . En Venezuela, se han realizado diferentes estudios que reportan resultados similares a los obtenidos en la presente investigación, en estados centro occidentales (Yaracuy y Carabobo) en donde se ha evidenciado que existe asociación significativa entre individuos positivos a T. cruzi y el tipo de vivienda no consolidada (rancho) y consolidada (casa), siendo en todos los casos los ranchos quienes tienen el mayor porcentaje de seropositivos 5,29 .

De la misma manera, se demostró que existe una asociación significativa entre la infección por T. cruzi y haber vivido en viviendas rurales con paredes y techos de palma o bahareque. Este tipo de material presenta fisuras que ofrecen un microclima ideal para la domiciliación de los triatominos, ya que le ofrecen las condiciones ideales de temperatura y humedad para un ciclo domiciliario ${ }^{30}$. El uso de palmeras como material de construcción de la vivienda permite el establecimiento de colonias de triatominos intradomiciliares, lo cual favorece la transmisión cuando éstos llegan infectados o encuentran animales domésticos igualmente infectados con T. cruzi 31.

En la presente investigación, la presencia de depósitos de herramientas o materiales y los anexos de bahareque en el peridomicilio resultó asociado a un mayor riesgo de adquirir la infección por $T$. cruzi, en las comunidades rurales estudiadas. Los depósitos de herramientas son estructuras en el peridomicilio, hechas con materiales deficientes o de baja calidad, sin servicios básicos, los cuales se usan como almacén. Este tipo de construcciones y los anexos de bahareque representan hábitats, donde los triatominos encuentran fuentes de alimento y refugio, permitiendo su proliferación y supervivencia 9 .

La presencia de aves dentro de las viviendas se asoció a la infección por T. cruzi en las comunidades rurales estudiadas. Se ha demostrado que la presencia de aves y perros en el domicilio está asociado a la seropositividad en humanos 32 . La presencia de aves (gallinas) en el domicilio constituye una fuente de alimento para los triatominos, en consecuencia aumenta la densidad poblacional de estos en las viviendas, un mayor contacto de los vectores con los reservorios domésticos, incrementando la probabilidad de triatominos infectados y por ende de humanos positivos 33,34.

Otra de las variables estudiadas con asociación significativa para la infección por T. cruzi fue la presencia de leña en el peridomicilio de las viviendas. Los insectos triatominos pueden ser encontrados dentro de diferentes ecotopos, estos pueden dispersarse pasivamente de medios silvestres o selváticos a medios peridomésticos 33,34. Algunas especies asociadas a la madera en el ambiente natural, frecuentemente son introducidas en medios ambientes artificiales a través de la leña 35. Black et al. 36 en Ecuador demostraron que la leña es un factor de riesgo significativo que se encuentra asociado a la infección por T. cruzi en las comunidades endémicas estudiadas.

En la presente investigación, la personas mayores de 40 años presentaron mayor seroprevalencia, aun cuando muchas de estas personas actualmente viven en viviendas de bloques y cemento, antiguamente se domiciliaban en viviendas tipo rancho (paredes de bahareque, techos de palma y pisos de tierra), esto pudo haber sido uno de los factores de riesgo asociados a la infección a T. cruzi. Una de las limitaciones del estudio fue el tamaño de la muestra de las personas a evaluar, ya que el diseño mues- 
treal permitió sólo evaluar a los residentes de la vivienda seleccionada de forma aleatoria, en algunos casos en ésta únicamente residían una o dos personas.

Aunque en el presente trabajo se encontró una mayor seroprevalencia de la infección por T. cruzi, asociada a viviendas con precarias condiciones de construcción, también se detectaron seropositivos en viviendas bien consolidadas con paredes frisadas, pisos de cemento o baldosas y techos de zinc. Adicionalmente, como fue reportado previamente T. maculata es el principal vector en el estado, habiéndose adaptado a las viviendas en las poblaciones rurales del estado Sucre, lo cual demuestra que el patrón habitual de la transmisión de la infección por T. cruzi puede estar cambiando, ya que T. maculata, asociado principalmente a ambientes silvestres y del peridomicilio, se encuentra actualmente en un proceso de cambio, adaptándose a ambientes poco habituales para el establecimiento de su nicho ecológico 9,21. Las variables epidemiológicas asociadas a la infección por T. cruzi, reportadas en este estudio demuestran la dinámica de la transmisión de la enfermedad en la actualidad para las poblaciones rurales del estado y éstas hacen posible a la vez que se mantenga la cadena epidemiológica tanto del parásito, como de los vectores involucrados en la transmisión de la enfermedad. La detección de casos seropositivos en menores de 10 años revela transmisión vectorial activa, lo cual debe ser tomado en cuenta por el sistema de salud pública gubernamental como una alerta epidemiológica para que sean aplicadas las medidas de control respectivas.

\section{Colaboradores}

N. García-Jordán contribuyó con la realización de los ensayos, análisis de datos y escritura del manuscrito. M. Berrizbeitia contribuyó con la realización del análisis de datos, revisión crítica y aprobación final de la escritura del manuscrito. J. Rodríguez colaboró en la realización de las pruebas serológicas y revisión del manuscrito. J. L. Concepción contribuyó en la revisión crítica de la escritura del manuscrito. A. Cáceres y W. Quiñones colaboraron en la revisión del análisis de datos y revisión del manuscrito.

\section{Agradecimientos}

Los autores expresan su reconocimiento a todos los habitantes del estado Sucre que gentilmente permitieron la realización de este estudio, aportando los datos de las encuestas socioeconómicas y de las tomas de muestras. A los líderes comunitarios de cada población rural evaluada quienes apoyaron y ayudaron a todo el equipo de campo y al Centro de Asesorías y Proyectos Estadísticos, Escuela de Estadística, Universidad de Los Andes, Venezuela. Proyecto financiado por el Ministerio del Poder Popular para la Salud, Dirección de Investigación y Educación, Proyecto no 490029000 y por el Proyecto Misión Ciencia n 2007001425.

\section{Conflicto de intereses}

Los autores declaramos que no hay conflicto de intereses.

\section{Referencias}

1. Rassi A, Anis RJ, Marin-Neto JA. Chagas disease. Lancet 2010; 375:1388-402.

2. World Health Organization. Chagas disease in Latin America: an epidemiological update based on 2010 estimates. Wkly Epidemiol Rec 2015; 90:33-43.

3. Ministerio del Poder Popular para la Salud. Enfermedad de Chagas. Boletín Epidemiológico 2008; (7).

4. Briceño Z, Orlandoni G, Torres E, Mogollón A, Concepción J, Rodríguez C, et al. Factores de riesgo asociadas a la enfermedad Chagas en comunidades rurales en Lara, Venezuela. Rev Costarr Salud Pública 2014; 23:13-24.

5. Salazar J, Gallego L, Suárez B, Heredia H, Hernández T, Naranjo M. Estudio seroepidemiológico de la enfermedad de Chagas en la comunidad Copey-El Guayabillo, Estado Carabobo, Venezuela. Rev Cub Med Trop 2014; 66:34-47.

6. Cermeño J, Askew E, Salazar F. Seroprevalencia de la enfermedad de Chagas en comunidades indígenas de los estados Bolívar y Delta Macuro. Saber 2013; 25:373-81.

7. Devera R, Fleming B, Romero G, Blanco Y, Amaya I, Tutaya R, et al. Seronegatividad para la infección chagásica en la comunidad La Carolina, estado Bolívar, Venezuela. Saber 2014; 26:347-52.

8. Noya BA, Diaz-Bello Z, Colmenares C, RuizGuevara R, Mauriello L, Munoz-Calderon A, et al. Update on oral Chagas disease outbreaks in Venezuela: epidemiological, clinical and diagnostic approaches. Mem Inst Oswaldo Cruz 2015; 110:377-86. 
9. Bonfante-Cabarcas R, Rodríguez-Bonfante C, Vielma BO, García D, Saldivia AM, Aldana E, et al. Seroprevalencia de la infección por Trypanosoma cruzi y factores asociados en una área endémica de Venezuela. Cad Saúde Pública 2011; 27:1917-29.

10. Feliciangeli MD, Sanchez-Martin MJ, Suarez B, Marrero R, Torrellas A, Bravo A, et al. Risk factors for Trypanosoma cruzi human infection in Barinas State, Venezuela. Am J Trop Med Hyg 2007; 76:915-21.

11. Scheaffer R, Mendenhall W, Ott L. Elementos de muestreo. México DF: Grupo Editorial Iberoamericana; 2006.

12. Berrizbeitia M, Ndao M, Bubis J, Gottschalk M, Ache A, Lacouture S, et al. Field evaluation of four novel enzyme immunoassays for Chagas' disease in Venezuela blood banks: comparison of assays using fixed-epimastigotes, fixedtrypomastigotes or trypomastigote excreted-secreted antigens from two Trypanosoma cruzi strains. Transfus Med 2006; 16:419-31.

13. Guhl F, Nicholls S. Manual de procedimientos para el diagnóstico de la enfermedad de Chagas. Mérida: Universidad de los Andes; 2001.

14. Fletcher R, Fletcher, S, Wagner E. Epidemiología clínica. Aspectos fundamentales. 2a Ed. Barcelona: Elsevier Masson; 1998.

15. Añez N, Crisante G, Rojas A. Update on Chagas disease in Venezuela: a review. Mem Inst Oswaldo Cruz 2004; 99:781-7.

16. Berrizbeitia M, Moreno D, Ward BJ, Gómez E, Jorquera A, Rodríguez J, et al. Trypanosoma cruzi infection in an indigenous Kariña community in Eastern Venezuela. Epidemiol Res Int 2012; 2012:138259.

17. Berrizbeitia M, Ward BJ, Bubis J, Gottschalk M, Ache A, Perdomo D, et al. 85-kDa protein of Trypanosoma cruzi purified by affinity chromatography used in the multiple antigen binding assay (MABA) for the diagnosis of T. cruzi infection in a Venezuelan rural community. Parasitol Res 2010; 106:1127-34.

18. Berrizbeitia M, Aguilera G, Ward B, Rodríguez J, Jorquera A, Ndao M. Seroprevalencia de la infección por Trypanosoma cruzi en la población rural de Miraflores, estado Monagas. Estabilidad y diferencia de reactividad de epimastigotes fijados. Rev Soc Venez Microbiol 2010; 30:55-60.

19. Millán D, Kiriakos D, Sánchez E, Santana H. Seropositividad para la enfermedad de Chagas en una población rural del estado Anzoátegui. Inf Med 2006; 8:119-28.

20. Rodríguez-Bonfante C, Amaro A, García M, Wohlert LEM, Guillen P, García RA, et al. Epidemiología de la enfermedad de Chagas en el municipio Andrés Eloy Blanco, Lara, Venezuela: infestación triatomínica y seroprevalencia en humanos. Cad Saúde Pública 2007; 23:1133-40.

21. Garcia-Jordan N, Berrizbeitia M, Concepcion JL, Aldana E, Caceres A, Quinones W. Entomological study of Trypanosoma cruzi vectors in the rural communities of Sucre state, Venezuela. Biomedica 2015; 35:247-57.
22. Cazorla-Perfetti D, Nieves-Blanco E. Triatominos de Venezuela: aspectos taxonómicos, biológicos, distribución geográfica e importancia médica. Av Cardiol 2010; 30:347-69.

23. Feliciangeli MD, Carrasco H, Patterson JS, Suarez B, Martinez C, Medina M. Mixed domestic infestation by Rhodnius prolixus Stal, 1859 and Panstrongylus geniculatus Latreille, 1811, vector incrimination, and seroprevalence for Trypanosoma cruzi among inhabitants in El Guamito, Lara State, Venezuela. Am J Trop Med Hyg 2004; 71:501-5.

24. Traviezo L, Bonfante R. Estudio seroepidemiológico de la enfermedad de Chagas en la localidad de Caballito, municipio Simón Planas, estado Lara, Venezuela. Parasitol Latinoam 2004; 59:46-54.

25. Ache A, Matos AJ. Interrupting Chagas disease transmission in Venezuela. Rev Inst Med Trop São Paulo 2001; 43:37-43.

26. Vila MA. Aspectos geográficos del Estado Sucre. Caracas: CVF; 1965. (Serie Monografías Estadales)

27. de Casas S, Carcavallo R, Galíndez I, Burgos J. Bioclimatic factors and zones of life. In: Carcavallo R, Galíndez-Girón I, Jurberg J, Lent $\mathrm{H}$, editors. Atlas of Chagas disease vectors in the Americas. Rio de Janeiro: Editora Fiocruz; 1999. p. 747-92.

28. Briceño-León R. La enfermedad de Chagas en las Américas: una perspectiva de ecosalud. Cad Saúde Pública 2009; 25 Suppl 2:S71-82.

29. Castillo S, Álvarez C, Rodríguez-Bonfante C, Gil A, Bonfante-Cabarcas R, Loyo Y, et al. Seroprevalencia de Trypanosoma cruzi y factores de riesgo en comunidades rurales municipio Nirgua estadon Yaracuy. Boletín Médico de Postgrado 2004; XX:73-9.

30. Zeledon R, Rabinovich JE. Chagas' disease: an ecological appraisal with special emphasis on its insect vectors. Annu Rev Entomol 1981; 26:101-33.

31. Añez N, Crisante G, Rojas A, Diaz N, AñezRojas N, Carrasco H, et al. La cara oculta de la enfermedad de Chagas en Venezuela. Bol Malariol Salud Ambient 2003; XLIII:45-57.

32. Gurtler RE, Chuit R, Cecere MC, Castanera MB, Cohen JE, Segura EL. Household prevalence of seropositivity for Trypanosoma cruzi in three rural villages in northwest Argentina: environmental, demographic, and entomologic associations. Am J Trop Med Hyg 1998; 59:741-9.

33. Cecere MC, Gurtler RE, Chuit R, Cohen JE. Effects of chickens on the prevalence of infestation and population density of Triatoma infestans in rural houses of north-west Argentina. Med Vet Entomol 1997; 11:383-8.

34. Gurtler RE, Cohen JE, Cecere MC, Lauricella MA, Chuit R, Segura EL. Influence of humans and domestic animals on the household prevalence of Trypanosoma cruzi in Triatoma infestans populations in northwest Argentina. Am J Trop Med Hyg 1998; 58:748-58. 
35. Carrizo R, Pickenhayn J, Carrizo M. Chagas urbano en San Juan. Diagnóstico, revisión y propuesta para un sistema integrado de ataque. Rev Argent Cardiol 2008; 76:480-7.

\section{Abstract}

The current study aimed to determine the seroprevalence of Trypanosoma cruzi infection in Sucre State, Venezuela, and its association with epidemiological risk factors. The cluster sampling design allowed selecting 96 villages and 576 dwellings in the State's 15 municipalities. A total of 2,212 serum samples were analyzed by ELISA, HAI, and IFI. Seroprevalence in Sucre State was 3.12\%. Risk factors associated with T. cruzi infection were: accumulated garbage, flooring and wall materials, type of dwelling, living in a house with wattle and daub walls and/or straw roofing, living in a house with risky walls and roofing, risky buildings and wattle and daub outbuildings, poultry inside the human dwelling, and presence of firewood. Infection was associated with individual age, and three seropositive cases were found in individuals less than 15 years of age. Sucre State has epidemiological factors that favor the risk of acquiring T. cruzi infection.

Chagas Disease; Trypanosoma cruzi; Rural Population
36. Black CL, Ocana S, Riner D, Costales JA, Lascano MS, Davila S, et al. Household risk factors for Trypanosoma cruzi seropositivity in two geographic regions of Ecuador. J Parasitol 2007; 93:12-6.

\section{Resumo}

O estudo teve como objetivo determinar a soroprevalência da infecção pelo Trypanosoma cruzi no Estado de Sucre, Venezuela, e a associação com fatores de risco epidemiológicos. O delineamento da amostragem em clusters permitiu a seleção de 96 vilarejos e 576 moradias nos 15 municípios do Estado. No total, 2.212 amostras de soro foram analisadas com ELISA, HAI e IFI. O estudo mostrou uma soroprevalência de 3,12\% no Estado de Sucre. Os seguintes fatores de risco estiveram associados à infecção pelo T. cruzi: acúmulo de lixo, materiais de piso e paredes impróprios, tipo de moradia, moradias com paredes de pau-a-pique e/ou teto de palha, moradias em situação de risco e construções anexas feitas de pau-a-pique, aves dentro das moradias e presença de lenha. A infecção esteve associada à idade individual, e três casos soropositivos foram identificados em indivíduos com menos de 15 anos de idade. O Estado de Sucre apresenta fatores epidemiológicos que aumentam o risco de infecção pelo T. cruzi.

Doença de Chagas; Trypanosoma cruzi; População Rural
Recibido el 27/Mar/2016

Versión final presentada el 12/Nov/2016 Aprobado el 06/Ene/2017 